\section{Bernd Orzessek}

\section{Pulsation als Grundfunktion des Lebendigen}

Nach Wilhelm Reich [1] ist die Grundfunktion dieser Lebenskraft die Pulsation, also ein rhythmisches Expandieren und Kontrahieren. Diese Grundfunktion ist allem Lebendigen gemein, vom Kleinsten bis zum Grössten. Wenn wir nun aber eine solche periodische Pulsation verstehen und grafisch als eine Funktion der Zeit darstellen, dann erhalten wir das Abbild einer Sinusschwingung. Aus einer Vielzahl dieser Schwingungen, aus den kleinsten Bausteinen der Materie, über die verschiedenen Hierarchien der Zell- und Organebenen, eingebunden in externe Oszillatoren und Taktgeber circa- bis ultradianer Perioden, entsteht eine Matrix aus biologischen Frequenzen. Diese Matrix zeigt in ihrer Komplexität emergente Eigenschaften, harmonikale Ordnung und fraktale Strukturen.

Um diese Selbstregulation zu verstehen, müssen wir Abstand nehmen vom mechanistischen Konzept des Herzens als Pumpe: Wir beobachten das Blut in seiner Pulsation und seinem Strömen in Wirbelformen, noch bevor sich die Myokardzellen zu ihrem ersten Impuls synchronisieren.

Das Herz ist dann auch vielmehr ein Organ der Empfindung und der Regulation. Ungezählte biologische Schwingungen müssen in unseren Körpern zu einer Matrix höherer Ordnung zusammenfinden und suchen Erneuerung und Stabilität. Dies wird immer neu gewährleistet, mit jedem Pulsschlag unseres Herzens.

Diese Ergänzung des Herzschlages um die Erfordernisse der Anpassung an das Hier und Jetzt nennen wir Re-

\title{
Herzratenvariabilität
}

Die Analyse der Herzratenvariabilität (HRV) ist ein Fenster, das einen Blick auf den unmittelbaren Ausdruck lebensenergetischer Prozesse ermöglicht. Die HRV beschreibt die Veränderlichkeit der Herzfrequenz als die Variabilität der Länge konsekutiver RR-Intervalle.

gulation. Die Konstante dieser lebendigen Erinnerungsprozesse sind die stetigen Veränderungen. Ein Versagen dieser Regulationsfähigkeit lässt uns aus der Matrix fallen, die Anbindung verlieren. Diese Ordnungsstrukturen und seine Dynamik zu erfassen ist Aufgabe der HRV-Analyse.

\section{HRV-Analyse}

Die HRV ist alters- und geschlechtsabhängig, wird von genetischen Polymorphismen beeinflusst, von der Atmung massgeblich moduliert und ist von vielen internen (Emotionen, Stress, mentale Tätigkeit usw.) und externen Faktoren (Körperlage, Tageszeit, Geräuschpegel usw.) abhängig. Die verschiedenen Methoden einer Analyse der HRV lassen sich grob in folgende Bereiche einteilen: Time Domain, Frequency Domain, geometrische Verfahren und Methoden, die nichtlineare mathematische Modelle nutzen.

\section{Time Domain}

Neben anderen statistischen Parametern beschreibt die SDNN die Standardabweichung (engl. standard deviation) der HRV. RMSSD und pNN50 bilden den parasympathisch beeinflussten Anteil der HRV ab.

Typische statistische Berechnung der Time Domain:

- AVNN: Durchschnittswert aller RR-Intervalle.
- SDNN: Standardabweichung aller NN-Intervalle.

- SDANN: Standardabweichung des Durchschnitts der RR-Intervalle in allen 5-Minuten-Segmenten einer 24-Stunden-Messung.

- RMSSD: Die Quadratwurzel des Mittelwerts der Quadratwurzeln der Differenzen zwischen jeweils nachfolgenden RR-Intervallen.

- pNN50: Prozentzahl aller Differenzen zwischen nachfolgenden RR-Intervallen grösser als $50 \mathrm{~ms}$.

\section{Frequency Domain}

Diese Analysen wurden 1981 zunächst von Akselrod et al. [2] eingeführt. Grundlage dieser ist das Theorem des französischen Mathematikers Jean Baptiste Fourier, das es ermöglicht, in allen Systemen, die periodische Oszillationen zeigen, die darin enthaltenen Sinusschwingungen und ihre Frequenzen $\mathrm{zu}$ analysieren. Die sog. Power in $\mathrm{ms}^{2}$ eines gegebenen Frequenzbandes zeigt somit die Amplitude der Variabilität der RR-Intervalle als Schwingungen in diesem Frequenzband (Abb. 1).

Hier werden den beiden Frequenzbändern LF (Low Frequency; 0,04$0,15 \mathrm{~Hz}$ ) und HF (High Frequency; $0,15-0,4 \mathrm{~Hz}$ ) die grösste Aufmerksamkeit geschenkt. Im HF-Band finden wir als oszillatorische Komponente die respiratorische Sinusarrhythmie und die parasympathische Aktivität. Im LF-Band finden wir sympathische und parasympathische Einflüsse.

\section{KARGER}

Fax +497614520714 Information@Karger.d www.karger.com
(ㄱ) 2010 S. Karger GmbH, Freiburg

Accessible online at: www.karger.com/szg
Dr. med. Bernd Orzessek

Aeskulap-Klinik

Ärztliche Ganzheitsmedizin

Gersauerstrasse 8, 6440 Brunnen am Vierwaldstättersee, Schweiz

Tel. +41 41 82549-49, Fax -65

bernd.orzessek@aeskulap.com 
Über die Komponenten ULF (Ultra Low Frequency; nur Langzeitmessung) und VLF (Very Low Frequency; $0,0-0,04 \mathrm{~Hz}$ ) weiss man bis heute noch sehr wenig; man identifiziert Homöostaseregulationen wie die Thermoregulation und viele circadiane und ultradiane Körperrhythmen in diesem Bereich.

\section{Nichtlineare Auswertung}

In den vergangenen Jahren sind eine Anzahl nichtlinearer mathematischer Funktionen eingeführt worden; neben anderen beschreiben die Approximate Entropy (ApEn), die Komplexität und die Detrended Fluctuation Analysis (DFA) die Korrelation innerhalb eines Signals.

Die nichtlineare grafische Darstellung des Poincare Plots (Abb. 2) zeigt auf der Y-Achse das gegebene RRIntervall in ms und auf der X-Achse das diesem vorangegangene Intervall (RRn+1 vs. RRn).

Eine regelrechte Variabilität der Intervalle wird eine eiförmige Verteilung ergeben. Vorteile sind die Erkennbarkeit ektoper Ereignisse und die grafische Darstellung der Kurzzeitvariabilität SD1 (assoziiert mit der respiratorischen Sinusarrhythmie, RSA) und der Langzeitvariabilität SD2 (in Beziehung zur SDNN).

\section{Anwendung}

\section{Kurzzeit-HRV-Analyse}

Die Dauer der Messung wird häufig auf 5 min festgelegt. Es können alle oben aufgeführten Analysemethoden angewendet werden.

\section{4-h-HRV-Analyse}

Mittels einer ambulanten HolterEKG-Messung werden während einer typischen Tagesroutine die RR-Intervalle während $24 \mathrm{~h}$ aufgezeichnet und analysiert. Es entsteht ein sog. autochrones Bild, wenn eine Spektralanalyse auf der Y-Achse und die Zeit auf der X-Achse dargestellt werden. Diese
Abb. 1.

Spektrale Analyse.
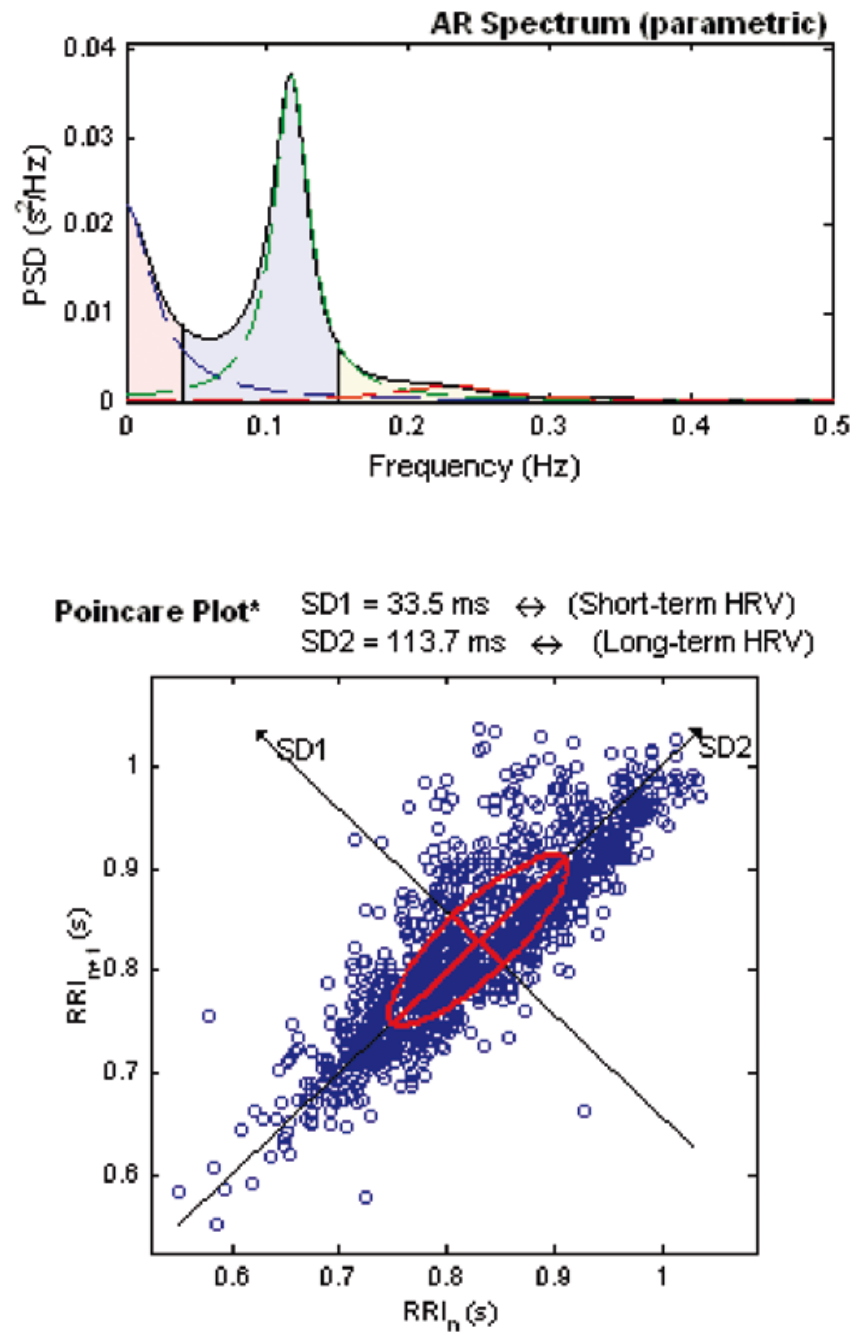

Abb. 2.

Poincare Plot.

${ }^{\times}$Calculated from the non-detrended selected RRI signal.
Methode erlaubt die Beurteilung von circadianen Rhythmen, Stress- und Erholungszeiten und auch der Schlafphasen.

Bewährt hat sich diese Methode bei uns bei der Therapieplanung chronisch erschöpfter Patienten, der Stresserkrankung und der chronischen Schmerzerkrankung mit der so häufigen Störung der Schlafarchitektur. Es können so nicht nur selbstschädigende Verhaltensweisen erkannt, sondern auch Ressourcen entdeckt und angesprochen werden.

\section{HRV-Biofeedback}

Damit ein komplexer vielzelliger Organismus auf einen Stimulus antworten kann, muss ein «Entrainment», eine Koordination der verschiedenen biologischen Oszillatoren in Form von
Zellen, Geweben und Organen zu einer funktionellen Einheit, gewährleistet werden. Eine dieser Frequenzen liegt mit circa $0,1 \mathrm{~Hz}$ im Bereich der Traube-Hering-Meyer(THM)-Oszillationen. Ludwig Traube konnte 1865 unterhalb der bekannten systolischdiastolischen Blutdruckschwankungen eine langwellige Oszillation mit einer Frequenz von 0,1 bis $0,17 \mathrm{~Hz}$ nachweisen, die der Baroreflexschleife entspricht. Die THM-Oszillationen sind ein wesentlicher Bestandteil der Homöostasis.

Wählt der Therapeut eine Atemfrequenz um $0,1 \mathrm{~Hz}$ (entspricht 6 Atemzügen/min), ist dies ein biologischer Rhythmus, der die Aktivität der Baroreflexschleife und somit Prozesse der Blutdruckregulation repräsentiert. Atmung und Pulskurve, beide in 
der Frequenz um 0,1 Hz, können dann in Kohärenz zur Blutdruckregulation gelangen.

Die Kohärenz der biologischen Oszillatoren Atmung, Herzfrequenz und Blutdruckregulation fördert die selbstorganisierenden Regelprozesse der Homöostasefindung.

Physiologische Effekte sind eine Erhöhung der parasympathischen Aktivität, eine Stimulation des Immunsystems gemessen am Immunglobulin A ( $\operatorname{Ig}$ A) im Speichel, eine Steigerung des Dehydroepiandrosterons (DHEA), eine Senkung des Blutdrucks und ein positiver Einfluss auf Emotionen und die emotionale Intelligenz.

Indikationen für das HRV-Biofeedback-Training sind: Arterielle Hypertonie, Stresserkrankung, chronische Schmerzsyndrome, Angsterkrankungen, Depression, Fibromyalgie, posttraumatische Belastungsstörung (PTSD), Herzinsuffizienz und Asthma bronchiale.

\section{HRV-Monitoring in Echtzeit}

Mit dieser Methode kann die sympathovagale Balance unter einer Intervention in Echtzeit kontrolliert und dokumentiert werden.

HRV-Analyse nach Alexander Riftine Mittels Power-Spektrum-Analyse und speziellen Algorithmen wird hier die Balance zwischen der Aktivität des sympathischen und parasympathischen Nervensystems in vier Quadranten grafisch dargestellt. Nach einer Messung in Ruhe wird unter Provokation durch Orthostase (Belastung) oder Taktatmung bzw. vasovagale Manöver (Testung der parasympathischen Reserve) eine zweite Messung aufgetragen und mit der ersten in Beziehung gesetzt.

Diese Analysemethode gewinnt an Bedeutung zum einen in der Therapiekontrolle, zum anderen bewährt sich diese als Eingangsuntersuchung zur Orientierung über den konstitutionellen und vegetativen Ist-Zustand, was eine individuelle initiale Therapieplanung ermöglicht.

In der grafischen Darstellung ist die parasympathische Aktivität in der horizontalen Achse aufgeführt, rechts im Positiven und links im Negativen, sowie die sympathische Aktivität in der vertikalen Achse, oben im Positiven und unten im Negativen. Der Normalbereich wurde im rechten oberen Quadranten identifiziert. Mit zunehmender vegetativer Erschöpfung (parasympathische Depression) wandert dieser Messwert immer mehr nach links in den negativen Messbereich des parasympathischen Systems, in der Praxis oft auch mit einem überschiessenden Anstieg des Sympathikotonus als Stressreaktion mit einem Messwert im linken oberen Quadranten.

Diesbezüglich ist in verschiedenen Studien eine positive Korrelation des LF/HF-Quotienten mit erhöhten Katecholaminen und der Speichelalpha-Amylase beschrieben worden.

Entsprechend lassen sich laborchemisch bei Burn-out-Patienten mit Messwerten im linken unteren Quadranten gelegentlich niedrige KortisolProfile und pathologisch erniedrigte DHEA-Werte messen.

Nachdem erst kürzlich Lin et al. [3] den Yin-Mangel bei Krebspatienten mit typischen HRV-Befunden assoziieren konnten, regen wir auch an, in der klinischen Befunddiskussion die horizontale Achse mit Yin und die vertikale Achse mit Yang zu belegen. Die Regulationsstarren stellen sich dann als starre Linie im Rhythmogramm bzw. als Blockade der symphathovagalen Balance unter Belastung dar.

\section{Sonifikation der HRV (HerzMusik)}

Grundlage einer jeden HerzMusik ist zunächst die Aufnahme der RR-Intervalle mittels EKG-Sensoren. Diese Daten werden nun von einer Software formatiert und die Schwingungsmuster analysiert, skaliert und orchestral sonifiziert. Es entsteht dann ohne wei- teren kompositorischen Einfluss von aussen eine akustische Abbildung der in der HRV enthaltenen Informationen, die wir als Musik wahrnehmen.

Wenn wir nun unter Therapie das vegetative Schwingungsmuster des Patienten gefördert haben, diesen hochkomplexen vegetativen Zustand mittels einer HRV-Messung aufnehmen und uns diese Informationen dann als Audio-CD vorliegen, können wir später über das Hören mit diesen dynamischen Mustern ein Resonanzsystem herstellen und uns wieder $\mathrm{zu}$ mehr Komplexität verhelfen.

Ein weiterer möglicher Link zwischen der akustischen Information und dem Schwingungssystem unseres Körpers, der eine Resonanzbildung ermöglichen könnte, sind die akustischen Spiegelneuronen, die Keysers und Gazzola [4] im September 2006 erstmals nachwiesen.

Die Real-Time-Sonifikation ermöglicht therapeutische Prozesse im Sinne eines Biofeedback, während die Musik des Herzens in Echtzeit von jedem Herzschlag neu komponiert wird. Der Patient wird sich so seiner vegetativen Dynamik bewusst und lernt diese anzunehmen bzw. zu beeinflussen.

In der Real-Time-Sonifikation können auch zwei Patienten zugleich ihre HerzMusik erklingen lassen (HerzHarmonik); dies wird genutzt für Kohärenz- und Empathietraining, z.B. in der Partnerschaft, zur Herstellung einer therapeutischen Resonanz und auch als Kommunikationshilfe, z.B. zwischen Mutter und autistischem Kind.

Aber warum erkennen wir in der HRV Musik? Wir stellen diesbezüglich die Hypothese auf, dass in beiden die gleichen der Natur immanenten Ordnungsprinzipien wirken.

So wie die Schöpfung in lebendiger Mathematik ihre Ordnung sucht, Sonnenblumenkerne gemäss der Fibonacci-Reihe angeordnet sind, Da Vinci den goldenen Schnitt in unserer menschlichen Anatomie allerorts wiederfand 
und Küstenlinien fraktaler Gestaltung folgen, so finden wir auch in der HRV die Mathematik als Ausdruck schöpferischer Ordnungsprinzipien.

Mathematik und Geometrie sind aber auch die Grundlage unserer $\mathrm{Mu}$ sik. Wir empfinden diese als heilsam, tröstend und schön, weil sie Ausdruck der Ordnung unseres Schöpfers sind. Über die Musik klingen also jene Ordnungsprinzipien der Schöpfung in uns an, in deren Matrix eingebettet wir uns entwickeln.

Eine besondere Form der HerzMusik ist die der Ungeborenen. Das Ungeborene befindet sich ja noch ganz eingebettet in das komplexe Schwingungssystem der Mutter und wächst und entwickelt sich in diese Matrix aus Rhythmus und Klang hinein.

Die HerzMusik wird in verschiedenen Settings aufgenommen und therapeutisch genutzt: Eine HerzMusik kann zunächst für einen einzelnen Patienten aufgenommen werden. Für die HerzMusik der Ungeborenen werden die RR-Intervalle von Mutter und ungeborenem Kind gleichzeitig registriert; es werden ihnen in der Sonifikation dann verschiedene Instrumente zugeteilt und es entsteht eine Musik, die von Mutter und Kind komponiert wurde. Die Audio-CD wird dann zur Geburtsvorbereitung genutzt.

Zusätzlich ist auch die Bildung eines Resonanzsystems über das elektromagnetische Feld des Herzens möglich; somit befände sich das Ungeborene ununterbrochen in einem solchen Informationsfeld seiner Mutter. Und folgerichtig hören wir auch beide eine gemeinsame Musik spielen. Aus dieser Resonanz erhält das noch unreife Schwingungssystem des Ungeborenen Stabilität, Energie und Information.

\section{Klinische Bedeutung der HRV}

Die Diagnostik der HRV etabliert sich als wichtige Methode zum Verständ- nis der Bindeglieder zwischen Soma und Psyche, zwischen Biologie und Verhalten. Nach Mück-Weymann [5] ist die HRV möglicherweise ein «Globalindikator für die Schwingungsfähigkeit (Resonanzfähigkeit) und Adaptivität bio-psycho-sozialer Funktionskreise im Austausch zwischen Organismus und Umwelt».

Nach der Regulationsstarre mit rigider Herzfrequenz bzw. fehlender pulsatiler energetischer Funktion stellt sich eine Erschöpfung der parasympathischen Leistung als Ausdruck einer vagalen Depression immer mehr als ein Hauptfaktor in der Ätiologie verschiedenster Pathologien heraus. Eine solche Starre und vagale Depression wird für ein breites Spektrum von Erkrankungen diagnostiziert; besonders gut belegt ist dies für die koronare Herzerkrankung betreffend Mortalität und Morbidität sowie für den plötzlichen Herztod.

In Übereinstimmung mit den vorausgegangenen Erläuterungen des energetischen Konzepts der rhythmischen Pulsation finden wir bei der Depression eine starre HRV, also mangelnde Schwingungsfähigkeit von Soma und Psyche als Einheit, ganz im Sinne Wilhelm Reichs. Des Weiteren besteht ein Mangel an parasympathischer Aktivität sowie Komplexität.

Auch die Krebserkrankungsrate steigt mit einer starren HRV. Wesentliches hierzu konnten Gimzewsky et al. $[6,7]$ im Rahmen ihrer Forschung zur Sonozytologie aufzeigen. Es gelang dieser Forschungsgruppe, Krebszellen von gesunden Zellen anhand ihres Klanges $\mathrm{zu}$ unterscheiden: Krebszellen rauschten, während die gesunden Zellen einen klaren Ton von sich gaben.

Therapieformen, für die eine Steigerung der parasympathischen Aktivität beobachtet werden konnte, sind Atemtherapie, energetisches Heilen, Akupunktur, Aufenthalt im Orgonakkumulator, Colon-Hydro-Therapie, HerzMusik, ionisierter Sauerstoff, körperliche Bewegung, Meditation, Seinszustände von Liebe und Dankbarkeit.

\section{Resonanz}

Resonanz ist das, «was die Welt zusammenhält» [8]. Der Organismus ist aus unzähligen Biorhythmen zusammengesetzt, die miteinander in Resonanz stehen müssen, damit er als ein Ganzes gegenüber der Aussenwelt in seiner Individualität besteht und Informationen sinnvoll weitergegeben sowie Gedächtnis, Lernen und Entwicklung möglich werden können.

Die Natur sucht die Resonanz, um miteinander in Kontakt zu treten. Zwei liebend verbundene Menschen zeigen eine Resonanz in der Ordnungsstruktur der spektralen Analyse der HRV über die räumliche Distanz hinweg. Ein ebensolches Phänomen kann in einer therapeutischen Beziehung stattfinden und ist möglicherweise Grundlage eines Heilungsprozesses.

\section{Literatur}

1 Reich R: The Discovery of the Orgone, Vol. 1: The Function of the Orgasm. New York, Orgone Institute Press, 1942, Chapter VII, 6.

2 Akselrod S, et al.: Power spectrum analysis of heart rate fluctuation: a quantitative probe of beat-to-beat cardiovascular control. Science 1981;213:220-222.

3 Lin SC, et al.: Severity of Yin deficiency syndrome and autonomic nervous system function in cancer patients. J Altern Complement Med 2009;15:87-91.

4 Gazzola V, Aziz-Zadeh L, Keysers C: Empathy and the somatotopic auditory mirror system in humans. Curr Biol 2006;16:1824-1829.

5 Mück-Weymann M: Was verrät die Herzratenvariabilität? www.hrv24.de/HRV-Einfuehrung. htm, Absatz 5.

6 Pelling AE, et al.: Local nanomechanical motion of the cell wall of Saccharomyces cerevisiae. Science 2004;305:1147-1150.

7 Roosth S: Screaming Yeast, Sonocytology, Cytoplasmic Milieus, and Cellular Subjectivities. MIT Working Paper. http://web.mit.edu/ sts/pubs/pdfs/MIT_STS_WorkingPaper_40_ Roosth.pdf.

8 Cramer F: Symphonie des Lebendigen - Versuch einer allgemeinen Resonanztheorie. Frankfurt/M., Insel, 1996. 